SORBY LECTURE July, 2009

\title{
CHARACTERISTICS OF EXFOLIATION CORROSION AT WELDMENTS IN AN Al-Zn-Mg ALLOY
}

\author{
C. Bagnall* \\ *MCS Associates, Inc., 637 Donohoe Road, Latrobe, PA 15650
}

\section{Introduction}

Exfoliation corrosion has been observed primarily in $\mathrm{Al}-\mathrm{Cu}$ and $\mathrm{Al}-\mathrm{Zn}-\mathrm{Mg}$ alloys. It is a unique manifestation of environmental attack, and is characterized by surface eruption of flakes, or the uplifting of layers of metal, notably in the heat affected zone region of weldments. The phenomenon is illustrated in this extended abstract. Effects on an Al-Zn-Mg alloy are shown after exposure of rolled plate with and without a weld to a standard acid salt spray test.

In the presentation to be made at the M\&M Conference in July, effects of cold work, in sheet and plate base metal, and precipitation hardening heat treatment are explored together with other physical and chemical factors that influence the initiation, growth and severity of exfoliation behavior. Investigations of exfoliation by other workers will be reviewed and some concluding statements will be made, in regard to underlying mechanisms and material conditions that promote this form of corrosion.

The main objective in this work was to investigate the heat affected zone response of MIG and TIG weldments to exfoliation. Welds were made with a range of parameters and used to join $\mathrm{Al}-4.5 \mathrm{Zn}, 1.8 \mathrm{Mg}, 0.7 \mathrm{Mn}$ material in solution-treated (T4) and agehardened (T6) conditions. In addition, susceptibility of extruded stock and un-welded rolled sheet, all in the T4 condition, were also studied.

\section{Experimental}

A standard acid salt spray test was employed. This entailed suspending specimens $(25 \mathrm{~mm}$ wide $\mathrm{x} 250 \mathrm{~mm}$ long) by a nylon cord in a fog chamber. A $5 \%$ sodium chloride solution with $0.6 \%$ glacial acetic acid was used. The $\mathrm{pH}$ was measured at $2.8+/$ 0.15 at a temperature of $35^{\circ} \mathrm{C}$, and atomized through a jet under an air pressure of 9 Newtons $/ \mathrm{cm}^{2}$. All specimens were exposed for a standard period of seven days. After exposure, they were washed in warm water, dipped in concentrated nitric acid for ten seconds, washed in cold water and finally dried with acetone.

\section{Results}

Exfoliation was observed on un-welded and welded material. Severity and characteristics were influenced by heat treated condition, the weld and weld parameters. Typical features of attack are illustrated in Figures 1 and 2.

In Figure 1(a), exfoliation on un-welded $10 \mathrm{~mm}$ thick plate in the T4 condition is shown. Layers of plate have lifted up from the exposed surface, like pages from a book. In Fig. 1(b), a side view of $10 \mathrm{~mm}$ plate with a four-pass TIG weld is shown. Formation of exfoliation in the heat-affected zone is a dominant feature. At the outer bounds of the HAZ, there is a band that is now resistant to attack. Beyond this, in the area unaffected by the weld thermal cycle, severe attack on the T4 plate propagates, as shown in (a). 


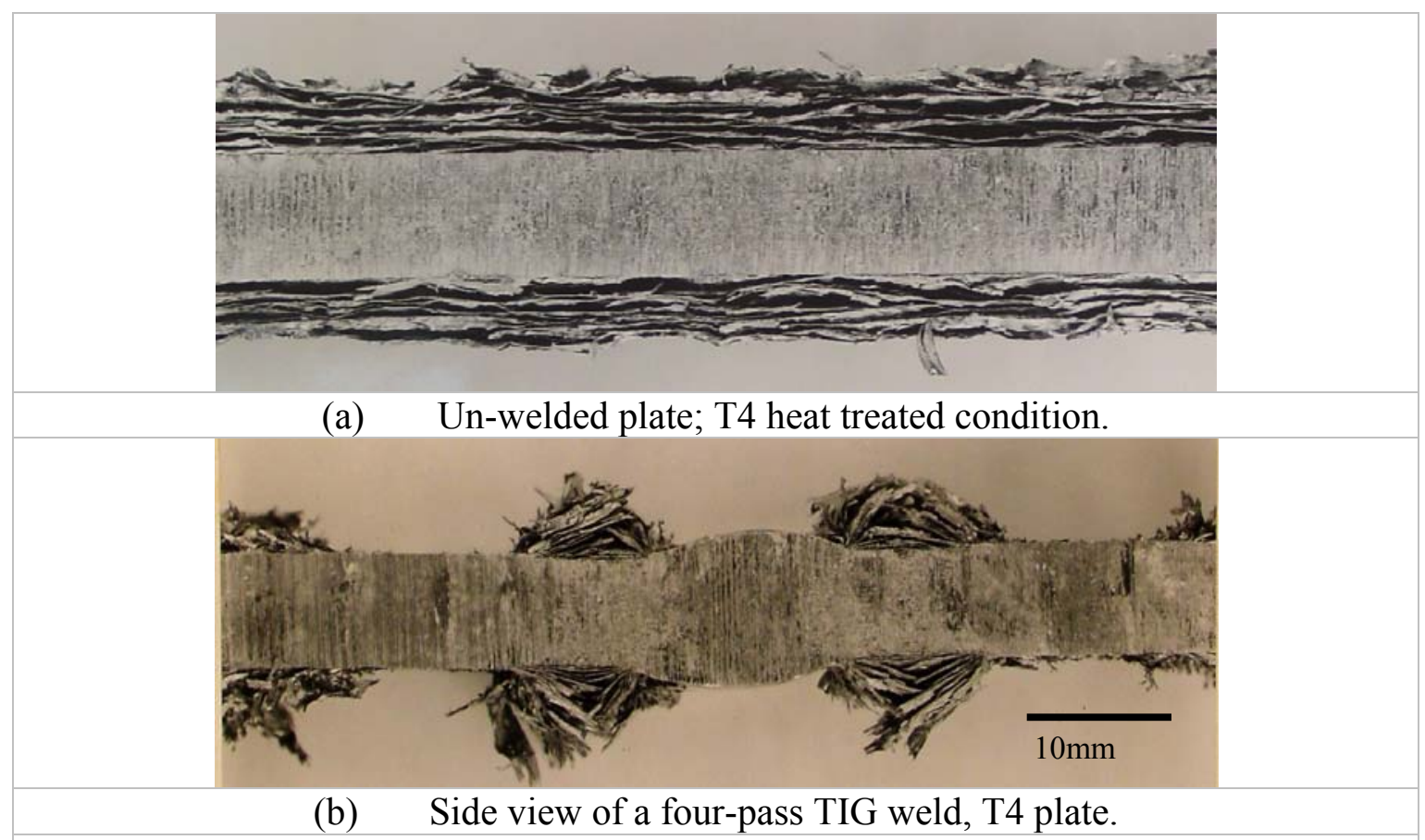

Figure1. Effect of a seven-day salt-spray test on $10 \mathrm{~mm}$ plate with and without a weld.

Figure 2 illustrates etched and unetched microstructures of a welded plate crosssection, showing the lifting of strips of metal. Propagation is along grain boundaries, and it can be concluded from the curved, spring-like exfoliated leaves that residual stress is an important component in the propagation mechanism.

Features associated with initiation and propagation of exfoliation, together with physical and chemical factors that either mitigate or exacerbate the phenomenon will be described in the presentation. One important finding from this work was that exfoliation can be prevented in this alloy by post-weld aging.

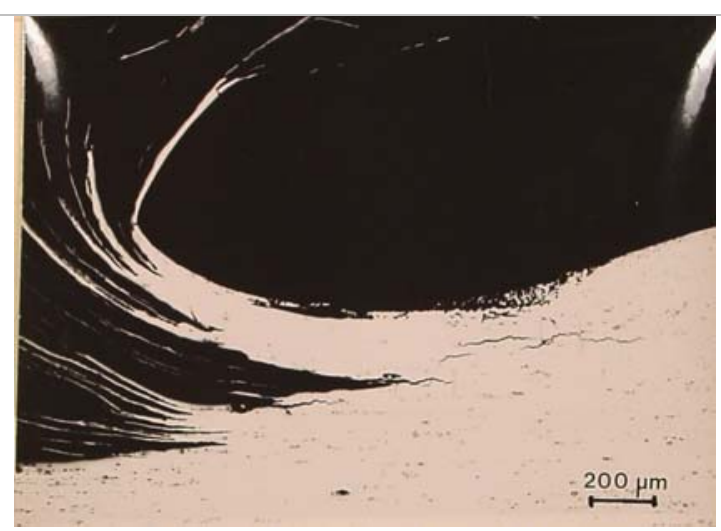

(a) As-polished transverse section

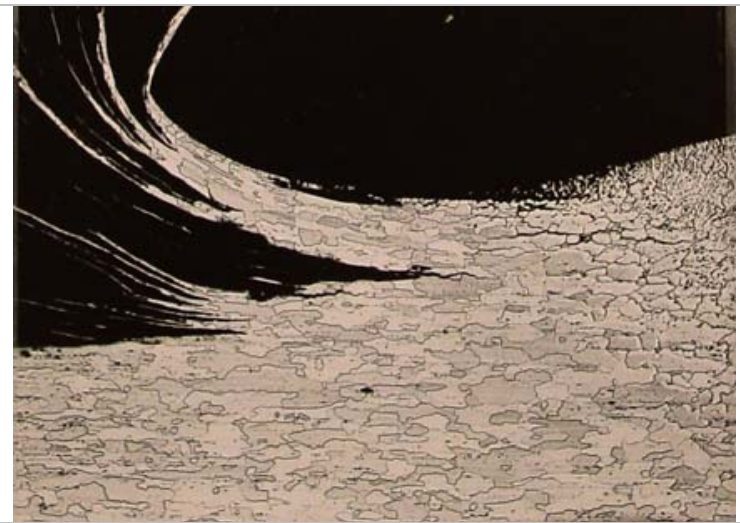

(b) Etched microstructure, showing intergranular propagation.

Figure 2. Detail of exfoliation growth adjacent to the fourth pass of a TIG weld, $10 \mathrm{~mm}$ plate 